\title{
Generalized wall functions and their application for simulation of turbulent flows
}

\author{
S. V. Utyuzhnikov ${ }^{*} \dagger$ \\ Department of Power, Propulsion \& Aerospace Engineering, School of Engineering, Cranfield University, \\ Cranfield, MK43 OAL, U.K.
}

\begin{abstract}
SUMMARY
Generalized wall functions in application to high-Reynolds-number turbulence models are derived. The wall functions are based on transfer of a boundary condition from a wall to some intermediate boundary near the wall (usually the first nearest to a wall mesh point but that is not obligatory). The boundary conditions on the intermediate boundary are of Robin-type and represented in a differential form. The wall functions are obtained in an analytical easy-to-implement form, taking into account source terms such as pressure gradient, and do not include free parameters. The log-profile assumption is not used in this approach. Although the generalized wall functions are obtained for the $k-\varepsilon$ model, generalization to other turbulence models is straightforward. The general approach suggested can be applied for studying high-temperature regimes with variable laminar viscosity and density. Copyright (c) 2005 John Wiley \& Sons, Ltd.
\end{abstract}

KEY WORDS: wall functions; turbulence; high-Reynolds-number model; $k-\varepsilon$ model

\section{INTRODUCTION}

The numerical simulation of the turbulent flows near walls is required in many industrial applications. It is well known that turbulence vanishes near a wall due to the no-slip boundary condition for the velocity as well as the blocking effect caused by the wall. In the vicinity of the wall, there is a thin sublayer with predominantly molecular diffusion. The sublayer has a substantial influence upon the remaining part of the flow. An adequate numerical resolution of a solution in the sublayer requires a very fine mesh because of the thinness of the sublayer and high gradients of the solution. Calculations are time consuming and may be impractical

\footnotetext{
*Correspondence to: S. V. Utyuzhnikov, Department of Power, Propulsion \& Aerospace Engineering, School of Engineering, Cranfield University, Cranfiled, MK43 0AL, U.K.

†E-mail: s.v.utyuzhnikov@cranfield.ac.uk

Contract/grant sponsor: FLOMANIA

Contract/grant sponsor: European Union; contract/grant number: G4RD-CT2001-00613
}

Received 27 April 2004

Revised 23 November 2004

Copyright (c) 2005 John Wiley \& Sons, Ltd. Accepted 25 November 2004 
for industrial applications. Because of the low-turbulent-Reynolds number in the sublayer, models that resolve the sublayer are called low-Reynolds-number (LR) models.

To remove the computational burden, high-Reynolds-number models (HR) have been developed that do not require resolution of the sublayer. It significantly saves computational efforts [1]. In the HR models, the boundary conditions or near-wall profiles are represented by wall functions. In most cases, the wall functions are semi-empirical and have very limited applications [1-5]. The wall functions were originally based on the log-law profile for the velocity $[2,3]$. The main disadvantage of these wall functions is a strong dependence on the location of the mesh point closest to the wall where the wall functions are applied. Such a problem is especially pronounced if the first mesh point is located inside the viscous sublayer. To avoid this, the scalable wall function approach is suggested in Reference [6]. Wilcox [7] showed that the pressure gradient must be taken into account to avoid the mesh dependence. In more recent approaches $[1,4,5]$ attempts have been made to take into account the pressure gradient and other effects such as buoyancy forces [1,5]. Numerical comparisons presented in References $[1,4,5]$ showed that such advanced wall functions give substantially better prediction than the standard wall functions. A brief review of wall functions can be found in, e.g. Reference [1]. In Reference [5], analytical wall functions are obtained by integrating boundary-layer-type equations in the vicinity of a wall using the assumption that all terms besides the diffusive one are constant. At the wall, the boundary conditions are the same as those used in the LR models. The analytical profiles for the effective viscosity are then used in the cell nearest to the wall to reconstruct the near-wall solution. The wall functions for the turbulent kinetic energy and its dissipation are based on a local approximate analytical solution for the velocity in the near-wall cell. Similar to the standard wall functions, this approach faces substantial problems if the nearest cell to the wall is in the viscous sublayer. Also, it is important to note that the second cell to the wall cannot be much smaller or bigger than the first one because of the integration over the first cell.

In the following sections, the method of boundary condition transfer is used [8]. The method allows us to transfer a boundary condition from a wall to some intermediate surface. It is shown that it is possible to transfer a boundary condition either approximately (analytically) or exactly (numerically). The boundary conditions on the intermediate surface are always of Robin-type and represented in a differential form. They can take into account the influence of the source terms in governing equations. These boundary conditions are interpreted as generalized wall functions. The location of the point, to which the boundary conditions are transferred, does not make any considerable effect on the mesh distribution nearby this point. The wall functions can be easily implemented.

The general approach suggested is applicable to studying high-temperature turbulent flows with variable density and laminar viscosity.

\section{MODEL EQUATION}

First, let us consider the following model equation:

$$
\left(\mu u_{y}\right)_{y}=R_{h}
$$

defined in a domain $\Omega=\left[0, y_{\mathrm{e}}\right]$ with Dirichlet boundary conditions

$$
u(0)=u_{0}, \quad u\left(y_{\mathrm{e}}\right)=u_{1}
$$


Equation (1) represents the general form of a boundary-layer-type equation. The right-hand side $R_{h}$ may contain the evaluation of source terms such as the pressure gradient, convective term or buoyancy force [9]. In many problems the contribution of the convective term in the wall function is not substantial and does not even exceed the error inevitable in its evaluation.

It is possible to transfer the boundary conditions from 0 to some point $y_{*}$ exactly [8]. Then, the boundary condition becomes of Robin-type:

$$
u\left(y_{*}\right)=u_{0}+f_{1} \frac{\mathrm{d} u}{\mathrm{~d} y}\left(y_{*}\right)-\left(\int_{0}^{y_{*}} R_{h} \mathrm{~d} y\right) \frac{f_{2}}{y_{*} \mu_{*}}
$$

where

$$
f_{1}=\int_{0}^{y_{*}} \frac{\mu\left(y_{*}\right)}{\mu(y)} \mathrm{d} y, \quad f_{2}=\int_{0}^{y_{*}} \frac{\mu\left(y_{*}\right)}{\mu(y)}\left(1-\frac{\int_{0}^{y} R_{h} \mathrm{~d} y}{\int_{0}^{y_{*}} R_{h} \mathrm{~d} y}\right) \mathrm{d} y
$$

Assuming that the coefficient varies piecewise linearly

$$
\mu= \begin{cases}\mu_{w} & \text { if } 0 \leqslant y \leqslant y_{v} \\ \mu_{w}+\left(\mu_{*}-\mu_{w}\right) \frac{y-y_{v}}{y_{*}-y_{v}} & \text { if } y_{v} \leqslant y \leqslant y_{*}\end{cases}
$$

where index $w$ means a value at $y=0$, it is possible to obtain analytical expressions for $f_{1}$ and $f_{2}$ if $R_{h}=$ const and $y_{v} \leqslant y_{*}$

$$
f_{1}=\alpha_{\mu} y_{v}\left(1+\theta \ln \alpha_{\mu}\right), \quad f_{2}=\alpha_{\mu} y_{v}\left[(1-\theta) y_{*}+y_{v}\left(\theta^{2} \alpha_{\mu} \ln \alpha_{\mu}-1 / 2+\theta\right)\right]
$$

where $\alpha_{\mu}=\mu_{*} / \mu_{w}, \theta^{-1}=\left(\mu_{*}-\mu_{w} / \mu_{w}\right)\left(y_{v} / y_{*}-y_{v}\right)$.

\section{GENERALIZED WALL FUNCTIONS}

We apply the method of boundary condition transfer given above to derive the generalized wall functions for the tangential velocity component $U$ or temperature $T$, and the turbulent kinetic energy $k$. Neglecting diffusion parallel to the wall, the appropriate transport equations can be written in form (1). Thus, the same approach is applied to all of these variables assuming that $R_{h}$ is variable in the case of the turbulent kinetic energy $k$ [9]. The boundary condition for $\varepsilon$ is the same as in Reference [5]. The effective viscosity $\mu_{\text {eff }}=\mu_{l}+\mu_{\mathrm{t}}$ can be evaluated via piecewise linear function (5), where $\mu_{l}$ (corresponds to $\mu_{w}$ in (5)) is the laminar viscosity and $\mu_{\mathrm{t}}$ is the turbulent viscosity. The sublayer thickness $y_{v}$ can be calculated as in Reference [5]:

$$
y_{v}=10.8 \mu_{l} /\left(\rho \sqrt{k_{*}}\right)
$$

The coefficients $f_{1}$ and $f_{2}$ in the wall functions (3)-(6) depend on $y_{*}$ and $k_{*}$ only. The latter value is determined from the solution of the HR model at the boundary point $y_{*}$. Hence, the intermediate boundary conditions (3) at $y=y_{*}$ complete the boundary-value problem in the interval $\left[y_{*}, y_{\mathrm{e}}\right]\left(y_{\mathrm{e}}\right.$ is the external boundary of the computational domain) and can be considered as generalized wall functions. These boundary conditions are of Robin-type and similar to the 'slip boundary condition' at the edge of the Knudsen-layer in aerodynamics. 
It is also important to note that boundary condition (3) is linear, therefore, it does not require iterations for its resolution. As it follows from (3), the source term in the wall functions can only be essential far enough from a wall because it is proportional to $y_{*}$ squared.

Upon obtaining a HR solution, it can be extended to the interval $\left[y_{v}, y_{*}\right]$ analytically [9]. It means that the intermediate boundary $y_{*}$ is not necessarily to be related to the nearest to the wall cell. It is possible to take $y_{*}$ far enough from the wall and, then, complement the solution on the region $\left[y_{v}, y_{*}\right]$.

The generalized wall functions obtained and their implementations are not based on a numerical integration in the inner region $\left[0, y_{*}\right]$, as in References $[1,4]$; therefore, the location of the intermediate boundary $y_{*}$ is not very substantial for the mesh distribution in the bulk domain. It means we can choose, e.g. a fine mesh despite a relatively big value $y_{*}$ (or vice versa) without losing stability.

\section{TEST CASE}

A fully developed plane channel flow has been considered as a test case. The flow is simulated far enough from the edge of the channel, so that the problem can be considered as 1D [10]. The standard HR $k-\varepsilon$ model has been used to test the wall function approach.

Here, $y$ is the distance to the wall, $p_{x}$ is the pressure gradient in the channel which is assumed to be negative, and $v=\mu / \rho$.

In the computations given below the Reynolds number is $R e=u_{\tau} h / v=395$, where $u_{\tau}=\sqrt{-h p_{x} / \rho}$ is the friction velocity, $h$ is half of the channel height. The dependence of the dimensionless velocity, $u^{+}=U / u_{\tau}$, on the universal coordinate, $y^{+}=y u_{\tau} / v$, is calculated using the approach developed in this work and compared against the benchmark results.

As it follows from Section 3, the generalized wall functions have only one free parameter which is the coordinate $y_{*}$ of the point to which the boundary conditions are moved. In Figure 1 the velocity profiles $u^{+}$obtained by the wall function approach are given against $y_{*}^{+}=y u_{\tau} / v$ for different values of $y_{*}$. The profiles are compared against the Reichardt's profile [11] representing the benchmark solution.

A comparison between several different kinds of wall functions is shown in Figure 2 for $y_{*}^{+}=100$. The dashed line corresponds to the homogeneous boundary conditions where the right-hand side $R_{h}$ in (3) is not taken into account. In this case, $f_{2}=0$ in (3). The error is significant. This confirms the importance of taking into consideration the source terms in the wall functions in the case of large values of $y_{*}$. If the source term is included in the boundary conditions for the velocity only, the prediction becomes much more accurate (dashdotted line). At the same time, the replacement of the boundary condition (3) for the turbulent kinetic energy $k$ by a frequently used boundary condition gives a substantial deviation from the benchmark solution (dotted line). The standard wall functions used for all variables [10] result in much better prediction (dash-double-dotted line). Yet, one can note that the channel flow is one of the most convenient test cases for the standard wall functions. The current approach gives more accurate predictions, as shown in Figure 1. The same conclusion was obtained in the case of $R e=3950$. The main advantages of the wall functions developed here are expected to be even more impressive when more complicated cases are considered. The generalized wall-functions have been recently implemented for the impinging jet test case $[12,13]$ and have given reasonably good results. Finally, it is important to emphasize 


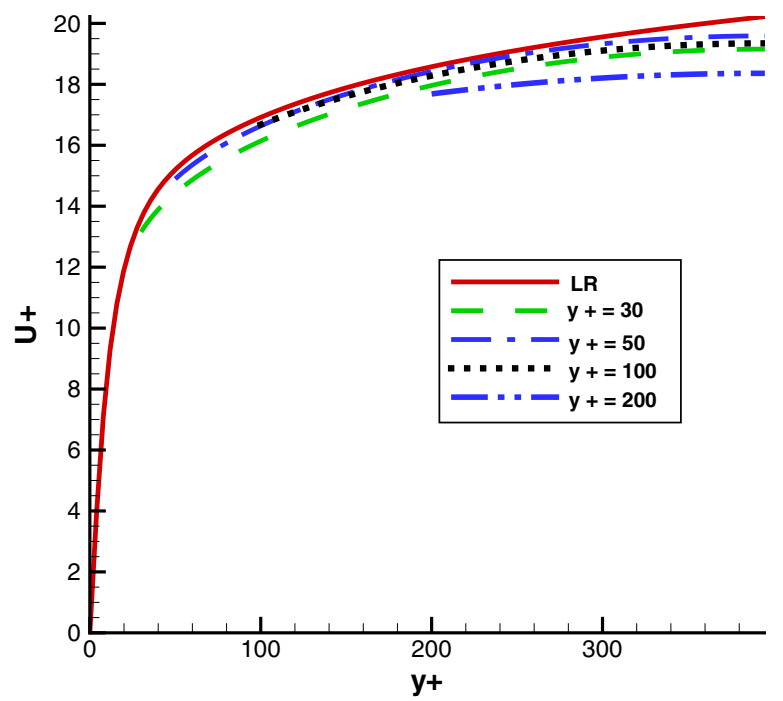

Figure 1. Velocity profile in channel flow. Solid line is Reichardt's profile; the other lines correspond to $y_{*}^{+}=30 ; 50 ; 100 ; 200$.

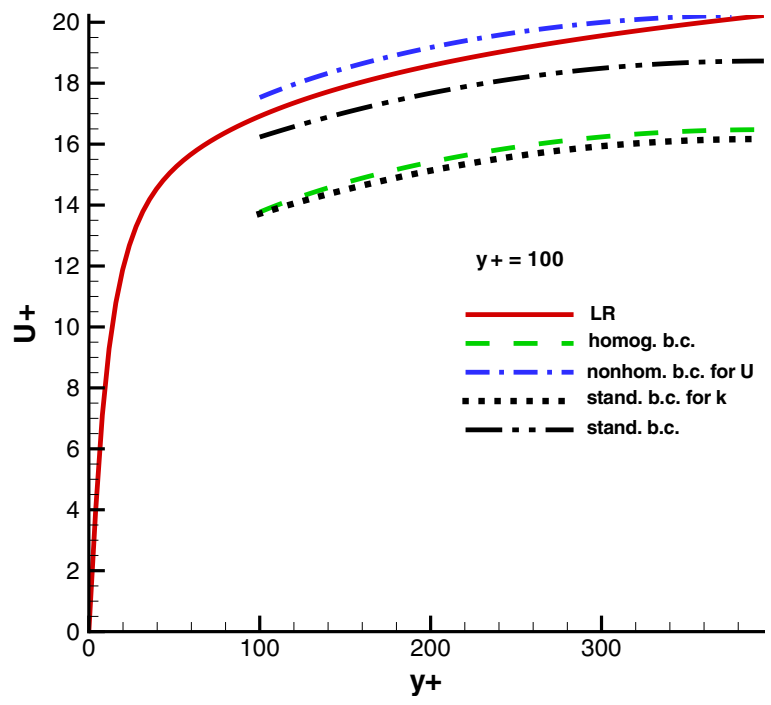

Figure 2. Velocity profile in channel flow with different wall-functions. Solid line is Reichardt's profile; the other lines correspond to homogeneous wall-functions for $U$ and $k$ (dashed line), and only for $k$ (dashed-dotted line); 'standard' wall-function for $k$ (dotted line) and for all variables (dashed-double-dotted line). 
that in contrast to the standard wall functions the approach in question is not based on the velocity-log-profile assumption or any similar additional information to match the solution.

\section{CONCLUSION}

Generalized wall functions have been developed. They are based on the transfer of boundary conditions from a wall to some point in the computational domain (usually the nearest to the wall grid point). The boundary conditions at this point are of Robin-type and represented in a differential form. These boundary conditions are interpreted as generalized wall functions taking into account source terms and used for HR models. The wall functions have been obtained in a compact easy-to-implement analytical form and they do not include any adjustable parameters. Testing this approach along with the $k-\varepsilon$ equations applied to a fully developed turbulent flow in a channel showed that the proposed wall functions are quite accurate even if the boundary conditions are set at a point either in a viscous sublayer or far beyond. The mesh distribution inside the computational domain can be chosen independent of the location of the intermediate boundary.

\section{ACKNOWLEDGEMENTS}

The author is grateful to A. V. Gerasimov and D. R. Laurence for fruitful discussions.

This work has been partially supported by the FLOMANIA project (Flow Physics ModellingAn Integrated Approach) a collaboration between Alenia, AEA, Bombardier, Dassault, EADS-CASA, EADS-Military Aircraft, EDF, NUMECA, DLR, FOI, IMFT, ONERA, Chalmers University, Imperial College, TU Berlin, UMIST and St. Petersburg State Technical University. The project is funded by the European Union and administrated by the CEC, Research Directorate-General, Growth Programme, under Contract no. G4RD-CT2001-00613.

\section{REFERENCES}

1. Craft TJ, Gant SE, Gerasimov AV, Iacovides H, Launder BE. Wall-function strategies for use in turbulent flow CFD. Proceedings of 12th International Heat Transfer Conference, Grenoble, France, 2002; 1-12.

2. Patankar SV, Spalding DB. Heat and Mass Transfer in Boundary Layers. Morgan-Grampian Press: London, 1967.

3. Launder BE, Spalding BI. The numerical computation of turbulent flows. Computer Methods in Applied Mechanics and Engineering 1974; 3:269-289.

4. Craft TJ, Gant SE, Iacovides H, Launder BE. Development and application of a numerical wall-function strategy for complex near-wall flows. Proceedings of ECCOMAS CFD 2001 Conference, Swansea, U.K., 2001; 1-20.

5. Craft TJ, Gerasimov AV, Iacovides H, Launder BE. Progress in the generalization of wall-functions treatments. International Journal of Heat and Fluid Flow 2002; 23:148-160.

6. Grotjans H, Menter FR. Wall functions for industrial applications. In Proceedings of Computational Fluid Dynamics'98, ECCOMAS, 1(2), Papailiou KD (ed.). Wiley: Chichester, U.K., 1998; 1112-1117.

7. Wilcox DC. Wall matching, a rational alternative to wall functions. AIAA Paper 89-611, Reno, NV, 1989.

8. Utyuzhnikov SV. Some numerical methods in application to low-Reynolds-number turbulence models. Preprint, arXiv:physics/0308014 (http://xxx.arxiv.org/ftp/physics/papers/0308/0308014.pdf), August 2003.

9. Utyuzhnikov SV. Generalized wall-functions for high-Reynolds-number turbulence models. Preprint, arXiv:physics/0310109 (http://xxx.arxiv.org/ftp/physics/papers/0310/0310109.pdf), October 2003.

10. Wilcox DC. Turbulence Modeling for CFD (2nd edn). DCW Industries: Canada, 2000.

11. Hinze JO. Turbulence (2nd edn). McGraw-Hill: New York, 1975.

12. Baughn J, Hechanova A, Yan X. An experimental study of entrainment effects on the heat transfer from a flat surface to a heated circular impinging jet. Heat Transfer 1991; 111:1023-1025.

13. Yan X, Baughn JW, Mesbah M. The effects of Reynolds number on the heat transfer distribution from a flat plate to an impinging jet. $A S M E$ HTD 1992; 226:1-7.

14. Chien K-Y. Predictions of channel and boundary-layer flows with a low-Reynolds-number turbulent model. AIAA Journal 1982; 20:33-38. 